\title{
Bleeding management in remote environment: the use of fresh whole blood transfusion and lyophilised plasma
}

\author{
Bruno Sicard ${ }^{1}$, Frédéric Marouzé ${ }^{2}$, Céline Roche ${ }^{3}$, Mathieu Carron ${ }^{4}$, \\ Sylvain Ausset $^{3}$, Anne Sailliol ${ }^{3}$ \\ ${ }^{1} \mathrm{PMSm}$, France \\ ${ }^{2} \mathrm{CGG}$, France \\ ${ }^{3}$ Service de Santé des Armées, France \\ ${ }^{4}$ Centre Hospitalier Universitaire de Nice, France
}

\begin{abstract}
To mitigate medical risks in remote environments, the authors have implemented an innovative integrated medical support solution for bleeding management on board ships since 2013. Fresh whole blood transfusion (FWBT) and lyophilised plasma were put in place to address life threatening haemorrhages in maritime operations in the Arctic and Antarctica. The authors are illustrating the bleeding risks with an actual case occurring in Antarctica prior to the implementation of these procedures. They are presenting the different steps involved in the complex process of FWBT, from blood donors' qualifications to actual transfusions. The pros and cons of blood transfusion in extreme remote environment are discussed, including the training of health care professionals, equipment requirements, legal and ethical issues, decision making in complex blood group matching, medical benefits and risks.
\end{abstract}

(Int Marit Health 2016; 67, 2: 79-82)

Key words: remote medicine, bleeding management, transfusion, plasma

\section{INTRODUCTION}

In our maritime medical support experience in the Kara Sea, the north-eastern Barents Sea, and near the Antarctic Peninsula, air medevac was most of the time not possible and even if it was possible it required from 2 to 5 sailing days, this could have been delayed even more due to weather conditions, known to change very rapidly in the Arctic area or in the Drake Passage.

To mitigate medical risks in these challenging environments, the authors have implemented an innovative integrated medical support solution on board seismic vessels (oil and gas surveys) since 2013 and Expedition Cruise vessels since 2015.

Many medevacs are conducted for suspected emergencies when clinically in doubt or if further medical examination is needed. Therefore, we first emphasized the upgrade of diagnostic tools to help identify and anticipate onboard emergency cases. The diagnostic equipment, including mobile X-ray, portable ultrasound, mobile slit lamp, point of care automated laboratory systems, were used by a specifically trained medical team consisting of an emergency physician and a registered nurse backed up by an adapted 24/7 top side support ashore.

Therapeutic tools were also upgraded and the most dramatic and innovative improvement was the implementation of fresh whole blood transfusion (FWBT) and lyophilised plasma to address trauma-induced life threatening haemorrhages and slow uncontrolled bleedings of medical origin (mostly gastrointestinal). These procedures were the only alternative in a remote environment where there is no access to certified blood products, because maintaining a blood bank would be both operationally difficult due to the short shelf life of blood products, most of them would expire on board, and also unethical due to the scarcity of 
blood products in general. Kauvar et al. [1] reported an $87 \%$ to $94 \%$ wastage rate for Packed Red Blood Cells in Forward Surgical Teams actively engaged in support of combat operations, and we could expect a much higher wastage rate in commercial maritime operations.

\section{ILLUSTRATION: BLEEDING CASE IN ANTARCTICA}

On December $6^{\text {th }}, 2014$, on an expedition cruise vessel, sailing near the Antarctica Peninsula, a 30-year-old male seafarer was admitted to the ship hospital at 2.00 AM for feeling faint with brief loss of consciousness. He reported a history of peptic ulcer, no current medical treatment nor over the counter drug use; he consumed alcohol on a daily basis but did not smoke. He reported dizziness for the last 3 days, dull epigastric pain partially relieved by food intake and red blood in his stools.

While walking to the exam couch, he briefly fainted again with a 20 s loss of consciousness as observed by the medical team. Vital signs were within normal limits (blood pressure [BP] 100/80 mm Hg, heart rate [HR] 100 bpm, respiratory rate $[\mathrm{RR}] 18 \mathrm{bpm}$, and peripheral capillary oxygen saturation $\left[\mathrm{SpO}_{2}\right]$ 99\%), blood glucose $145 \mathrm{mg} / \mathrm{dL}$, haemoglobin $11 \mathrm{~g} / \mathrm{dL}$, exhaled breath was negative for alcohol, electrocardiogram within normal limits and the only clinical finding was red blood on digital rectal exam. A pantoprazole IV administration was initiated and the patient remained stable until 6.30 AM when he started to feel weaker with foul rectal bleeding and at 8.30 AM he fainted again with a $30 \mathrm{~s}$ loss of consciousness, his haemoglobin dropping to $8.7 \mathrm{~g} / \mathrm{dL}$, IV tranexamic acid was administered. Top side support was called and medevac was triggered. Initially no plane was available and the medevac was to be conducted by sailing back to Ushuaia, a 2 days trip, which was unlikely to bring back alive the patient without finding an effective way to stop the bleeding or compensate the loss of blood. Unexpectedly, a plane engaged on another medevac ashore was localized in a nearby Chilean station in King George Island and the patient, still stable (HR 120, BP 105/50), was transported by a zodiac ashore at 3.30 PM, transferred to an Argentinian $\mathrm{C} 130$ Hercules which took off at 4.30 PM, and landed in Rio Gallegos, Argentina, at 7.00 PM. Rushed to the local hospital, the patient decompensated in haemorrhagic shock and required endotracheal intubation, vasoactive amines were administered, haemoglobin had dropped to $7 \mathrm{~g} / \mathrm{dL}$. The patient was hospitalised in the intensive care unit, his acute bleeding peptic ulcer benefitted from the transfusion of 3 units of red blood cells, gastric endoscopy was performed and the patient was discharged a week later. If this miraculous medevac plane had not been there, chance of survival with a 2 days medevac by sea was very slim without blood products.

\section{FRESH WHOLE BLOOD TRANSFUSION AND LYOPHILISED PLASMA}

\section{THE RISKS}

Managing a severe bleeding without blood bank is indeed a critical challenge. Acute haemorrhage from trauma or slow uncontrolled bleeding will lead to death if the cause of bleeding is not managed properly and the blood loss not compensated when getting below vital threshold. Stopping a bleeding may require, depending on the cause, sophisticated techniques, from damage control surgery (i.e. spleen fracture, liver wound), colonoscopy or gastroscopy (i.e. gastric ulcer, diverticulosis, etc.) to embolisation (i.e. pelvic fracture, posterior epistaxis, etc.) and specialised healthcare providers who are not easily deployable in a remote setting.

Also, in some places (tropical, sub-tropical, low level of care), local hospitals are capable of providing blood products but with a level of safety in regard to infectious agents way below standards of high income or less exposed countries which implies to reconsider the benefit/risk ratio for local blood transfusion.

Finally, with the development of Expedition Cruises with thousands of passengers sailing in the Polar Regions, some of them on anticoagulant prescription, the risk of severe bleeding of medical origin is more potent.

Massive blood loss will deplete the platelets and clotting factors, causing more bleeding, this coagulopathy is aggravated by hypothermia and acidosis, commonly observed in shocks, which are part of the "trauma triad of death". Therefore when life threatening bleeding occurs, blood products are critical. For resuscitation cases requiring massive transfusion, FWB is also more potent than the combination of packed red blood cells (RBC), platelets and fresh frozen plasma (FFP), with a better outcome for the patient [2-4]. This excellent potency is most probably due to the perfect $1 / 1 / 1$ ratio for plasma, red cells and platelets, the low dose of conservative additives and anticoagulants compare to units of blood bank products, and not using large amounts of RBC older than 14 days since storage time affects quality of cells [5]. A $500 \mathrm{~mL}$ warm FWB unit provides all blood components, including coagulation factors, unaltered and in right proportion and temperature (haematocrit 38-50\%, 150,000-400,000 platelets per microliter, and 100\% activity of clotting factors) compare to the $660 \mathrm{~mL}$ of a mix of 1 unit of RBC, with 1 unit of FFP, and 1 unit of platelet concentrate with a whole haematocrit of $29 \%, 88,000$ platelets per microliter, and 65\% coagulation factor activity [1].

This "haemorrhage damage control" process was adapted from military medical practice. FWBT has been used extensively to resuscitate casualties in military conflicts since World War I, but was discontinued in the 1980's with the occurrence of human immunodeficiency virus (HIV). Prior to HIV, the balance of a low risk of transmission of an 
infectious agent (usually syphilis, hepatitis), often treatable and not deadly in short term, was overcome by the benefit of saving a dying bleeding patient.

\section{THE MITIGATION MEASURES}

Since the wars in Afghanistan and Iraq the FWBT process was implemented again with an upgraded screening technique which lowers the risk of transmission of an infectious agent, and as Kauvar et al. [1] stated "FWBT is convenient, safe, and effective in certain military situations". This onsite screening of collected FWB units with the use of rapid detection tests for HIV, HCV, and HBV was recommended by Spinella et al. [5] as a standard process to minimize the risk of infectious agent transmission.

According to the French Military Blood Transfusion Centre, the residual infectious risk of transmitting an infection is estimated at approximately 0.00005 per FWB unit in French Military overseas operations where FWBT is implemented. Indeed, at the time of collection, all French military volunteer donors undergo standard risk questionnaire screening and are tested for HIV, hepatitis B virus (HBV), and hepatitis $\mathrm{C}$ virus ( $\mathrm{HCV}$ ). The residual risk is for human lymphotropic virus (HTLV) because currently there is no screening test for this virus usable before transfusion. The questionnaire screening must eliminate the donors coming from endemic areas (Intertropical Africa, Caribbean, Guyana) if they have not been tested before for this virus (i.e. regular donor). Bacterial contamination of FWB is a risk under field conditions but is limited by short storage period - only $6 \mathrm{~h}$ at room temperature.

These risks must be compared with a life threatening bleeding when neither certified Blood Bank products are available nor effective control of bleeding through surgery, endoscopy or endovascular embolisation is possible, leading to certain death. Still, even with a beneficial survival balance, due to these residual risks, FWBT is not approved by Food and Drug Administration nor other Civilian Health agencies for routine use, but it is approved by NATO (STANAG 2939 edition 5, 2010 Minimum Requirements for Blood, Blood Donors, and Associated Equipment). It is implemented by waiver only when no other alternative is available to save the life of a critically bleeding patient and a record of the decision process is kept.

The drawback of FWBT is that it is safer to use matching $\mathrm{ABO}$ blood type, therefore it is important to recruit enough volunteers to cover the whole spectrum of ABO blood types.

To do so we organised among the oil and gas professional crews an agile and customised prescreening process of volunteer blood donors on board and at sea in order to facilitate the process by reducing the amount of time the volunteers are required to dedicate to FWB collection process.
The onboard prescreening protocol included the following steps:

- on board lectures to the whole crew to explain the benefits and the rationale of volunteering for FWBT in order to recruit as many volunteer blood donors as possible;

- on board medical interviews to screen for low-risk volunteer donors according to recognised medical consensus on Go and No Go parameters, based on medical history, exposure to infectious agents, etc.;

- on board blood sampling for medically screened volunteers with centrifugation of blood samples prior to shipping to a certified laboratory for biological screening and blood typing. Biological screening included test for HIV, HTLV, hepatitis B and C, and syphilis. Results were kept confidential and managed directly with patient and his/her family physician; occupational physician from the seismic company did not have access to these results. In our experience with these oil and gas industry vessels, $5 \%$ of the volunteer donors were ruled out through biological screening.

For the expedition cruise vessels we did not implement a prescreening of volunteers, since the pool of potential donors change almost every week with new passengers and also the size of the pool was much larger (400 vs. 60 in Oil and Gas vessels), which allowed us to have more choice in the qualification of volunteers.

When a life threatening bleeding occurs, and FWBT is needed, to qualify a unit of whole blood on site requires the following steps:

- identification of the patient blood type and obtaining authorisation for transfusion from the patient or his/ her relatives;

- selecting matching volunteer blood donors: from among the pool of prescreened volunteers, if any, (however former volunteers could decide, whenever they wanted, to withdraw from the volunteering list, there was no formal commitment) or through a public call for donors in cruise vessels. For the latest we set a priority order to select multiple donors based on potential infectious status: active blood donors with up to date Donor's Card were favoured, while crew who had higher risk behaviours (more frequent compared to the passenger population) or patient's relatives with potential bias in screening interview were not the preferred donors;

- medical interview with the donor to rule out potential risk (medications, acute infection, risk behaviours, etc.), even for the prescreened volunteers;

- blood collection and on site rapid biological control for HIV, hepatitis B and C and blood type;

- blood sampling for complete a posteriori biological qualification (to be conducted after shipping to the laboratory ashore); 
- final bedside blood type matching between the receiver and the FWB pouch collected.

The medical team was initially trained in the French Military Transfusion Centre, Centre de Transfusion Sanguine des Armées (CTSA) in Paris, which is a reference centre for FWBT. The team had to conduct mandatory onboard drills to practice the complex procedure for FWB collection which only gives $70-90$ min to have the first $0.5 \mathrm{~L}$ pouch of FWB qualified for transfusion.

The FWB units, as regulated by the French Joint Military Health Service in Instruction No. 509396/DEF/DCSSA/PC/ /BMA [6] can be stored at room temperature for $6 \mathrm{~h}$ and for $48 \mathrm{~h}$ at $+2 /+6^{\circ} \mathrm{C}$.

In order to manage the golden hour, waiting for the first unit of FWB to be collected and qualified, the medical team could rely on lyophilised plasma, a universal freeze dried plasma produced by CTSA and which requires less than $6 \mathrm{~min}$ [7] to get prepared (sterile water is mixed with dry plasma powder) and can help manage the initial hypovolaemia and coagulopathy. This product is perfectly adapted to austere environment it has a 2 year shelf life at room temperature, and is universally used for any blood type. Finally a specific hot line providing $24 / 7$ medical advice on FWBT, run by specialists in emergency blood transfusion from CTSA, was available, in complement to the standard 24/7 maritime medical top side support.

\section{CONCLUSIONS}

Managing autonomously a severe patient for hours or days require a very significant upgrade of medical resources. Health Authorities, Flag regulations, Company Policies rarely address such extreme medical conditions. Ethic and laws require for ship owners and company staff, to implement mitigation measures adapted to the risks. Nowadays, the available technology make these upgrades feasible and affordable, especially when life is at stake or more trivially operations are in balance. The authors demonstrated through 3 years of medical support of polar seismic surveys and polar expedition cruises that these upgrades are ethically, technically, and economically sound.

Seafarers and cruise passengers deserve a level of care as close as the standards they receive at home. Deployment of FWBT and lyophilised plasma help reduce the gap between the level of care ashore and in a remote environment with regard to bleeding management.

Potentially, pooling these medical resources between different operators sailing in the same area, through "regional medical coordination" would make sense from an economic point of view but also technically. Indeed, pooling volunteer blood donors from a larger group of people increase the probability of getting the right blood type for the casualty when he/she carries a rare blood type.

Finally, in some remote areas of the world such a medical facility might be the best equipped hospital also for the local population and may be an asset which could be generously shared with the ones in great need.

\section{REFERENCES}

1. Kauvar DS, Holcomb JB, Norris GC, Hess JR. Fresh whole blood transfusion: a controversial military practice. J Trauma 2006; 61: 181-184.

2. Spinella PC. Warm fresh whole blood transfusion for severe hemorrhage: U.S. military and potential civilian applications. Crit Care Med 2008; 36 (7 suppl.): S340-S345.

3. Fisher AD, Miles EA, Cap AP, Strandenes G, Kane SF. Tactical damage control resuscitation. Mil Med 2015; 180: 869-875.

4. Spinella PC, Perkins JG, Grathwohl KW, Beekley AC, Holcomb JB. Warm fresh whole blood is independently associated with improved survival for patients with combat-related traumatic injuries. J Trauma 2009; 66 (4 suppl.): S69-S76.

5. Spinella PC, Perkins JG, Grathwohl KW et al. Risks associated with fresh whole blood and red blood cell transfusions in a combat support hospital. Crit Care Med 2007; 35: 2576-2581.

6. Instruction No 509396/DEF/DCSSA/PC/BMA. Relevant to blood transfusion in exceptional situation. 07 August 2015, p. 13.

7. Sailliol A, Martinaud C, Cap AP et al. The evolving role of lyophilized plasma in remote damage control resuscitation in the French Armed Forces Health Service. Transfusion 2013;53 (1 suppl.): S65-S71. 